\begin{tabular}{|c|c|c|}
\hline Case Reports in & \multicolumn{2}{|c|}{ Case Rep Gastroenterol 2018;12:773-779 } \\
\hline Gastroenterology & $\begin{array}{l}\text { DOI: 10.1159/000495936 } \\
\text { Published online: January 4, } 2019\end{array}$ & $\begin{array}{l}\text { (c) } 2019 \text { The Author(s) } \\
\text { Published by S. Karger AG, Basel } \\
\text { www.karger.com/crg }\end{array}$ \\
\hline & $\begin{array}{l}\text { This article is licensed under th } \\
\text { International License (CC BY-NC) } \\
\text { Usage and distribution for commel }\end{array}$ & $\begin{array}{l}\text { nons Attribution-NonCommercial } 4.0 \\
\text { ger.com/Services/OpenAccessLicense). } \\
\text { uires written permission. }\end{array}$ \\
\hline
\end{tabular}

\title{
Adenosquamous Carcinoma of the Choledochus
}

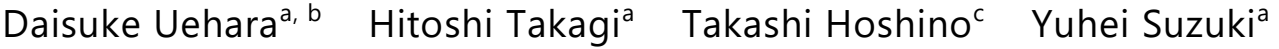 \\ Satoshi Takakusagi ${ }^{a}$ Kyoko Maruhashia Kazuko Kizawa ${ }^{a}$ \\ Takashi Kosone $^{a}$ Atsushi Naganuma $^{c}$ Etsuko Hisanagad ${ }^{d}$ Junko Hirato $^{d}$ \\ Satoru Kakizaki ${ }^{\text {b }}$ Toshio Uraoka ${ }^{b}$

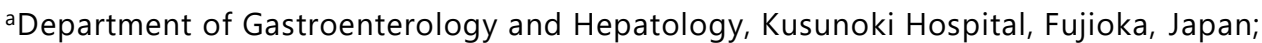 \\ bDepartment of Gastroenterology and Hepatology, Gunma University Graduate School of \\ Medicine, Maebashi, Japan; 'Department of Gastroenterology and Hepatology, Takasaki \\ General Medical Center, Takasaki, Japan; dDepartment of Pathology, Gunma University \\ Hospital, Maebashi, Japan
}

\section{Keywords}

Adenosquamous carcinoma $\cdot$ Choledochus cancer

\begin{abstract}
The patient was an 86-year-old man who was admitted with obstructive jaundice. Computed tomography revealed a tumor in the hilar choledochus with peripheral hepatic duct dilatation. Endoscopic cholangiography (ERC) demonstrated the defect in the choledochus. Brushing cytology during ERC showed Orange-G-philic keratinized atypical cells, which led to a diagnosis of squamous cell carcinoma. Chemotherapy with tegafur-gimeracil-oteracil potassium was ineffective and was discontinued due to adverse effects. The patient died 5 months after the diagnosis and autopsy revealed tubular adenocarcinoma of the hilar bile duct with squamous cell carcinoma component. Progression of the disease might influence the distribution of adenosquamous carcinoma. The clinicopathological sequence of adenosquamous carcinoma of the choledochus was documented.




\section{Introduction}

Adenosquamous carcinoma (ASC), which consists of adenocarcinoma and squamous cell carcinoma in one cancerous tissue, has been rarely reported [1]. Adenosquamous lung cancer is the most precisely described entity [1] because of its relative abundance, as it accounts for approximately $3 \%$ of lung cancer. Thus, bile duct ASC is rare [2,3]. The most common form of bile duct cancer is adenocarcinoma originating from the biliary epithelium. ASC of the biliary tract is reported to account for $0.6-2 \%$ of cases of bile duct cancer $[2,3]$. The diagnosis of the ASC before surgery or autopsy is difficult because biopsy specimens are not easily available. We herein report an advanced case of upper bile duct ASC and discuss the relevant literature.

\section{Case Report}

The patient was an 86-year-old man. His past history included laryngeal cancer at 70 years of age and appendectomy at 46 years of age. The patient was admitted to our hospital with dark urine and appetite loss, which had persisted for 1 week. A physical examination revealed the following: height, $158.1 \mathrm{~cm}$; body weight, $67.7 \mathrm{~kg}$; body mass index, $27.1 \mathrm{~kg} / \mathrm{m}^{2}$; blood pressure, 126/82 mm Hg; and a regular pulse ( 65 beats per minute). His consciousness was clear and his conjunctivae were icteric. Anemia or lymphadenopathy was not observed. No tumors were palpable around the body surface. The patient's laboratory data were follows: AST 629 IU/L; ALT 306 IU/L; ALP 4,030 U/mL; GGT 3,157 U/mL; and total bilirubin 5.15 $\mathrm{mg} / \mathrm{dL}$. The patient's tumor marker levels were elevated (CEA $22.4 \mathrm{ng} / \mathrm{mL}$; and CA19-9 1,192.6 U/L). SCC was within the normal range $(1.4 \mathrm{ng} / \mathrm{mL})$.

\section{The Clinical Course after Admission}

The patient was febrile with a body temperature of $37.5^{\circ} \mathrm{C}$ and developed jaundice with a total bilirubin level of $11.0 \mathrm{mg} / \mathrm{dL}$. Ultrasonography and computed tomography (Fig. 1a) revealed dilatation of the bilateral hepatic ducts with a mass lesion at the upper choledochus. Endoscopic retrograde cholangiography (ERC) revealed dilatation of the choledochus and a defect caused by the tumorous region in the upper choledochus (Fig. 1b). An endoscopic retrograde drainage tube was inserted through the papilla of Vater to improve the common bile duct obstruction.

Brushing cytology of the bile duct at ERC revealed atypical cells with irregular and large nuclei and Orange-G-philic keratinocytes on Papanicolau staining, which indicated the existence of squamous cells (Fig. 2a). Simultaneous biopsy of the bile duct showed erosion and destruction of the epithelium (Fig. 2b). The detached and atypical epithelium showed squamous differentiation. The specimen was positive for $\mathrm{p} 40$, a marker of squamous cells, and positive for $\mathrm{p63}$, another marker of squamous cells. The specimen was diffusely positive for CK19, which can detect biliary epithelium. The intraepithelial and interstitial invasion of inflammatory cells was observed. According to these findings, the patient was diagnosed with obstructive jaundice caused by squamous cell carcinoma arising from choledochus.

After the endoscopic retrograded drainage of the bile duct, the patient's jaundice disappeared, and palliative care was introduced with the administration of opioids. The patient's disease was diagnosed as stage IV (T2N1M1) and surgical treatment was not indicated. A single course of tegafur-gimeracil-oteracil potassium ( $50 \mathrm{mg} /$ body) was administered for 3 weeks; however, it was not continued due to appetite loss and the deterioration of the 
patient's performance status. Finally, the patient died due to cancer progression at 8 months after biliary drainage, without a recurrence of jaundice. An autopsy was performed.

\section{The Autopsy Findings}

Macroscopically, a massive tumor was observed surrounding the hepatic hilum (Fig. 3a). The endoscopic drainage tube inserted into the obstructed choledochus was still patent. The tumor was mostly adenocarcinoma with partial epidermoid differentiation with eosinophilic keratinocytes; squamous cells (detected based on p40 positivity) were present in a small part of the tumor (Fig. 3b). Metastasis was detected in the liver, lung, adrenal gland, and urinary bladder; metastases were dominant with adenocarcinoma. There was no recurrence of laryngeal cancer. Latent prostate cancer (moderately differentiated adenocarcinoma) was also present; thus, the patient suffered from triple cancer of the larynx, choledochus, and prostate.

\section{Discussion}

According to the WHO classification, ASC is defined by the presence of two malignant components: adenocarcinoma and squamous cell carcinoma [3]. A search of the PubMed database for reports on ASC in August 2018 yielded 3,328 papers. There were more than 100 reports on ASC of the lung (899), uterus (172), pancreas (162), breast (160), stomach (158), gall bladder (143), and colon (103). There were less than 100 reports on ASC of other sites, such as the head and neck, and anus. There were 76 reported cases of ASC of the bile duct. Okabayashi et al. [4] summarized the Japanese cases of biliary ASC and compared them with cases of adenocarcinoma in patients recruited for surgery with curative intent. The 5-year survival rate of patients with biliary ASC was $16 \%$, while that of patients with biliary adenocarcinoma was $23-44 \%$ [5]. The survival rate seemed to be lower for inoperable ASC such as the present case, which seemed to be more frequent.

The present case was diagnosed as squamous cell carcinoma based on cytology and biopsy; however, the squamous component was small at autopsy. The problematic point of this case was the discrepancy in the histology findings between the biopsy and autopsy. The biopsy specimen clearly showed squamous cell carcinoma, while the autopsy examination revealed adenocarcinoma-dominant choledochus cancer, with adenocarcinoma at metastatic sites. This may be explained by chemotherapy sensitivity or by the distribution of tumors at which biopsy was performed. Genomic approaches have revealed that ASC shows higher rates of TP3 mutation and 3p loss in comparison to adenocarcinoma [6].

At present, there is no specific strategy for the treatment of ASC because of its rarity. Cancer-directed surgery and a primary site of the ampulla of Vater may be the only favorable prognostic factors for patients with ASC of the bile duct [2]. The discrepancy of pathological findings at the relatively early stage and at autopsy might not be rare because of the characteristics of the cancer progression [7]. Further accumulation of cases of biliary ASC is mandatory to facilitate an early diagnosis and curative treatment.

\section{Statement of Ethics}

Written informed consent was obtained from the patient and his family for publication of this case report and any accompanying images. 


\section{Gastroenterology}

Uehara et al.: Adenosquamous Carcinoma of the Choledochus

\section{Disclosure Statement}

The authors have no conflict of interest.

\section{References}

1 Takamori S, Noguchi M, Morinaga S, Goya T, Tsugane S, Kakegawa T, et al. Clinicopathologic characteristics of adenosquamous carcinoma of the lung. Cancer. $1991 \mathrm{Feb} ; 67(3): 649-54$.

2 Qin BD, Jiao XD, Yuan LY, Liu K, Zang YS. Adenosquamous carcinoma of the bile duct: a population-based study. Cancer Manag Res. 2018 Mar;10:439-46.

3 Albores-Saavedra J, Scoazec JC, Wittekind C, et al. Tumours of the gallbladder and extrahepatic bile ducts. In: World Health Organization classification of tumours. Pathology \& genetics. Tumours of the digestive system. Lyon: IARC; 2000. p. 203-17.

4 Okabayashi T, Kobayashi M, Nishimori I, Namikawa T, Okamoto K, Onishi S, et al. Adenosquamous carcinoma of the extrahepatic biliary tract: clinicopathological analysis of Japanese cases of this uncommon disease. J Gastroenterol. 2005 Feb;40(2):192-9.

5 Miyakawa S, Ishihara S, Horiguchi A, Takada T, Miyazaki M, Nagakawa T. Biliary tract cancer treatment: 5,584 results from the Biliary Tract Cancer Statistics Registry from 1998 to 2004 in Japan. J Hepatobiliary Pancreat Surg. 2009;16(1):1-7.

6 Fang Y, Su Z, Xie J, Xue R, Ma Q, Li Y, et al. Genomic signatures of pancreatic adenosquamous carcinoma (PASC). J Pathol. 2017 Oct;243(2):155-9.

7 Grinberg LT, Ferraz da Silva LF, Galtarossa Xavier AC, Nascimento Saldiva PH, Mauad T. Clinico-pathological discrepancies in the diagnoses of solid malignancies. Pathol Res Pract. 2008;204(12):867-73. 

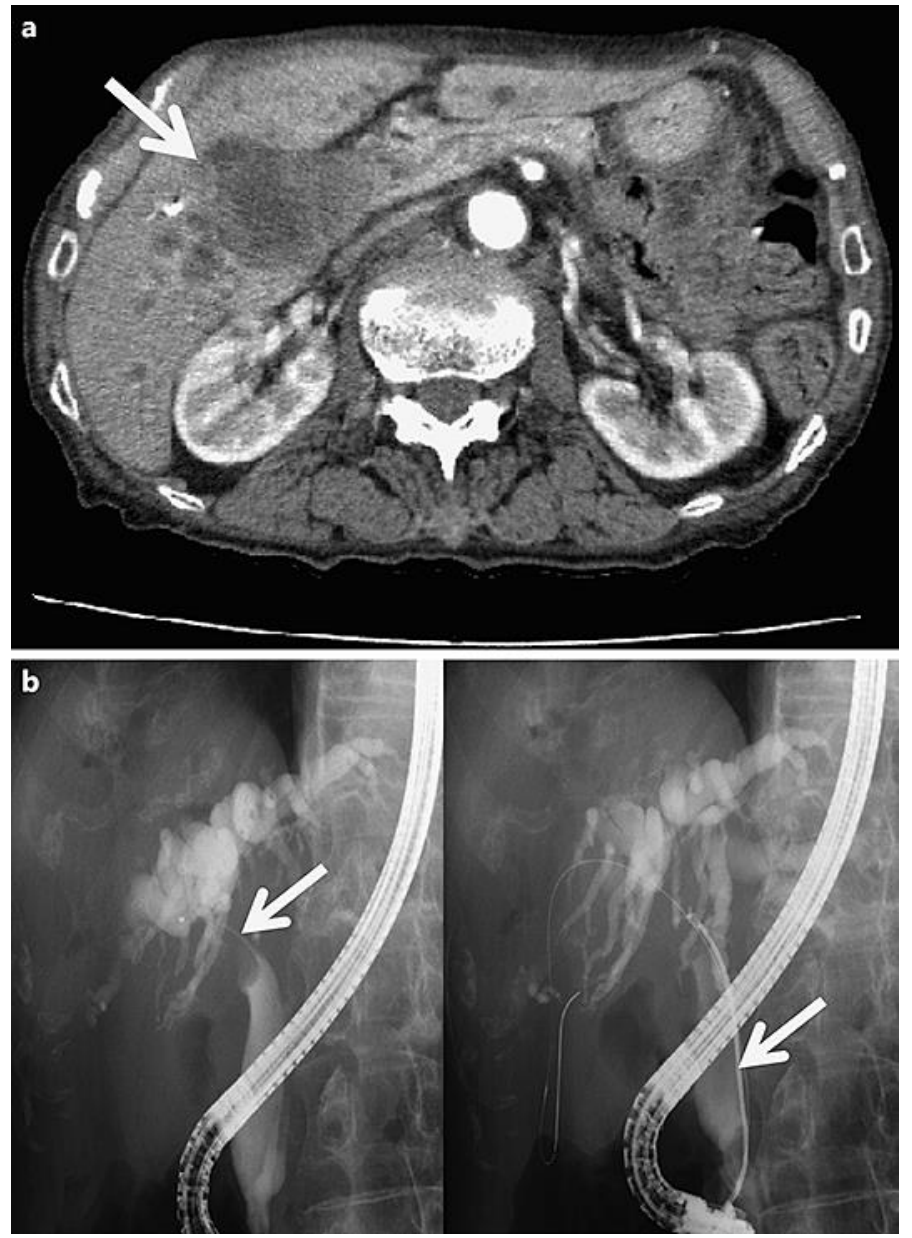

Fig. 1. a The abdominal computed tomography findings. A low-density area (arrow) was observed in the upper and hilar biliary tract accompanied by intrahepatic bile duct dilatation. b Left, The endoscopic retrograde cholangiography (ERC) findings. ERC revealed dilatation of the choledochus and a defect caused by the tumor in the upper choledochus (arrow). Choledochus biopsy and brushing cytology were performed. Right, an endoscopic retrograde drainage tube (arrow) was inserted through the papilla of Vater to improve the common bile duct obstruction. 

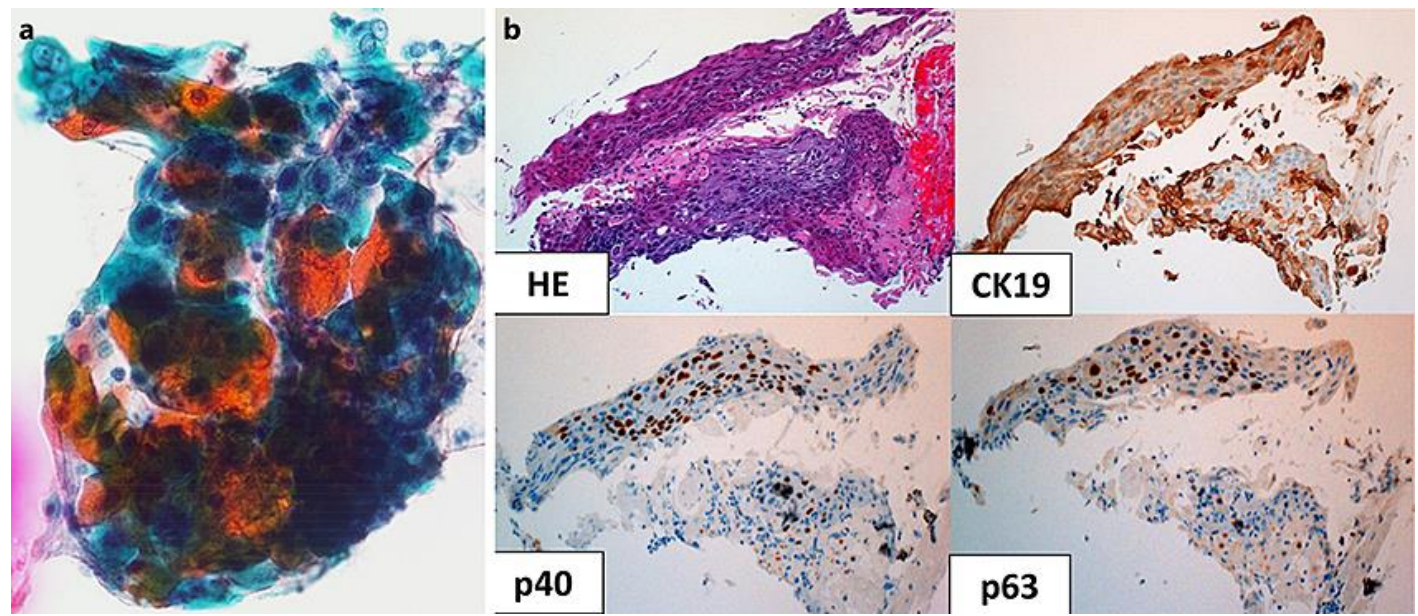

Fig. 2. The pathological findings. a Brushing cytology during ERC. Papanicolaou staining revealed atypical cells with irregular and large nuclei with Orange-G-philic keratinocytes, indicating the presence of squamous cells. b Choledochus biopsy during ERC. HE: hematoxylin and eosin staining showed erosion and destruction of the epithelium. The detached and atypical epithelium demonstrated squamous differentiation. CK19: the specimen was diffusely positive for CK19 (a biliary epithelium marker). p40: the specimen was positive for $\mathrm{p} 40$ (a squamous cell marker). p63: the specimen was also positive for p63 (a squamous cell marker). 


\section{Case Reports in Gastroenterology}
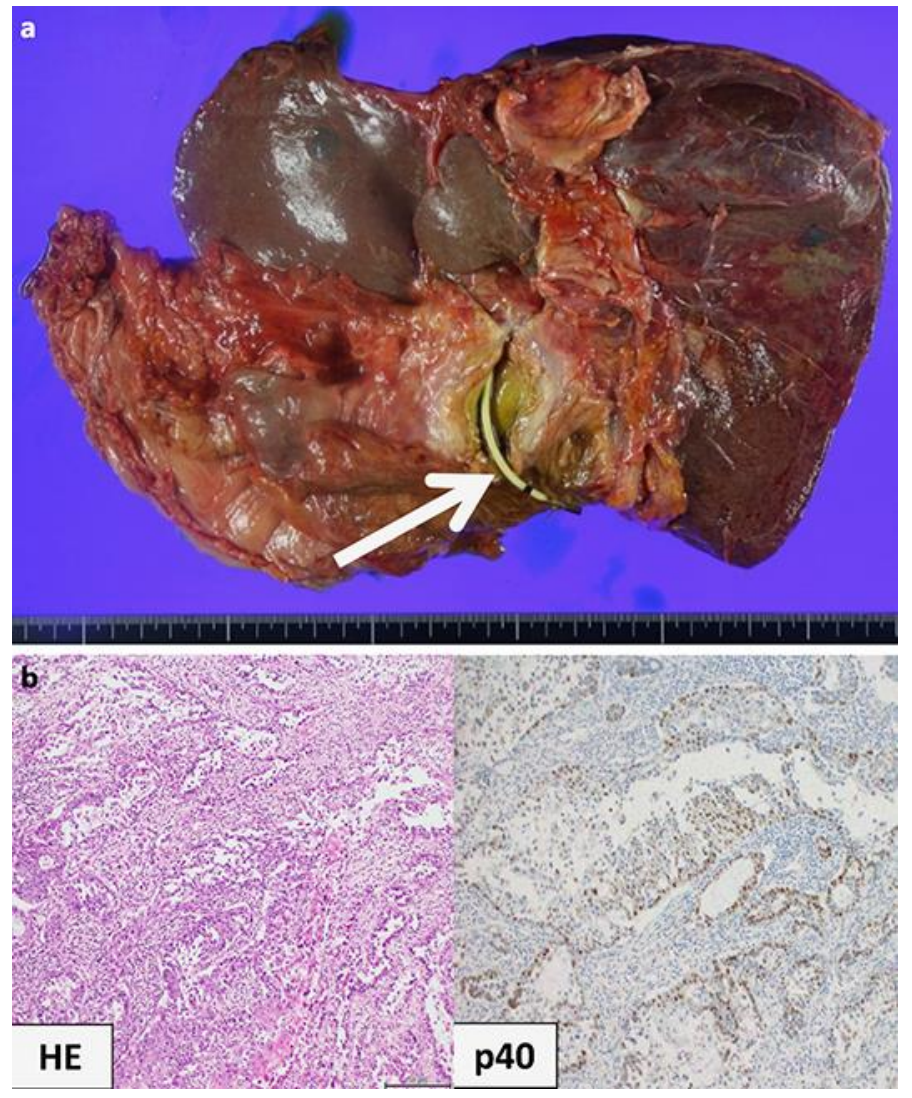

Fig. 3. The autopsy findings. a The macroscopic appearance of the choledochus tumor at autopsy. The ERBD tube (arrow) is patent and remained in the common bile duct surrounded by the tumor. $\mathbf{b}$ Autopsy revealed carcinomatous epithelial growth (left, HE, hematoxylin and eosin staining) and immunohistochemical staining using anti-p40 antibody was partially positive (right), indicating the presence of squamous cells. 